\title{
The verification of category and property statements
}

\author{
JAMES A. HAMPTON \\ The City University, London, England
}

\begin{abstract}
Two experiments compared the speed with which category and property statements of matched associative production frequency could be verified. The verification of category statements was consistently faster than that of property statements, for both true and false judgments. The category advantage did not interact with the level of production frequency for the true sentences. Implications for models of decision processes in semantic memory are discussed.
\end{abstract}

Experimental studies of semantic memory frequently have investigated the retrieval and verification of two kinds of proposition. The first kind, which is generally called a category proposition, specifies the class of things to which a concept belongs, as, for example, in "An apple is a fruit." The second kind, called a property proposition, describes attributes that are characteristics of the concept. An example of this kind would be "An apple has a peel." Although this distinction has been widely used, its theoretical basis is not well understood. Smith (1978) pointed out that there are no good logical criteria for differentiating category from property propositions. The two kinds of proposition are in fact intertranslatable. "An apple is a fruit" can be rephrased as "An apple has the property of fruitness," and "An apple has a peel" can be phrased as "An apple is in the class of peel-covered objects." It can thus be argued that the two types of proposition differ only in their syntactic form, and not in the form of their representation in memory. Therefore, other things being equal, whether or not the verification times for the two types of proposition differ is an interesting empirical question. Perhaps surprisingly, no direct comparison of the speed of verification of category and property statements under controlled conditions has been undertaken. The first aim of the present experiments was therefore to answer this simple empirical question.

Consideration of current models of semantic memory suggests that it is not easy to derive direct predictions for the outcome of this comparison. Network models (Glass \& Holyoak, 1975; Collins \& Loftus, 1975; Collins \& Quillian, 1969, 1972) assume that the major determinant of verification time for true semantic propositions is the ease of retrieving the information from the net-

The author acknowledges the help of John Gardiner and two anonymous reviewers, who made useful comments on an earlier draft of this paper, and of Frances Guy who helped with data collection. Requests for reprints should be addressed to the author at: Psychology Division, Department of Social Science and Humanities, The City University, Northampton Square, London EC1V 0HB, England. work, and that the most direct measure of this associative strength is production frequency in a generation task. As Johnson-Laird (1975) pointed out, this principle, originally known as Marbe's law of associations, accounts for a considerable number of experimental studies in which generation frequency later predicts retrieval speed (Conrad, 1972; Glass, Holyoak, \& O’Dell, 1974; Holyoak \& Glass, 1975; Wilkins, 1971). Thus, if associative frequency is held constant, then retrieval models in general would predict no difference in verification times for category and property predicates. Any such difference would therefore constitute an important departure from the generality of Marbe's law as an account of semantic memory processing times and would entail modifications to network models.

The alternative approach to semantic memory is the featural approach (Hampton, 1979, 1981; McCloskey \& Glucksberg, 1979; Schaeffer \& Wallace, 1970; Smith, Shoben, \& Rips, 1974). Rather than emphasizing retrieval processes, this approach assumes that category propositions are verified by a process of feature comparison. As with network models, it is not easy to derive a direct and obvious prediction from the models concerning the relative speed of verifying category and property information. On the one hand, one may suppose that, since property information generally consists of a single feature [e.g., COLOR (RED)], whereas category propositions depend on the evaluation of several features, property statements should be more rapidly verified. This proposal is consistent with Smith's (1978) suggestion that property statements are semantically more primitive than category statements (Wierzbicka, 1972). On the other hand, if features can be checked in parallel, or holistically (Smith et al., 1974), then with several features tending to confirm or contradict a category statement, but only one specific feature confirming or contradicting a property statement, category statements may be verified more quickly.

Network models might also be able to account for a difference in category and property verification times by adopting one of two suggestions contained in Collins 
and Quillian's (1969) early model. They found that category statements were verified faster than property statements (when nonnormative materials were used), which they explained by the suggestion that some properties must be retrieved via a category node. They proposed a principle of cognitive economy, whereby any property generally true of a class (such as "Birds can fly") would be stored at the category node for that class. Hence, "Canary can fly" would take longer to verify than "Canary is a bird," the latter being used to infer the former. On this particular account, category predicates should only be faster than properties that are also true of a superordinate class.

The second suggestion was that category nodes may be searched in series, whereas property nodes may be searched in parallel. This difference could obviously give rise to a difference in the overall speed of retrieving the two types of information. However, if one process is slower than the other, then, by this account, the effects of associative strength (as measured by production frequency-PF) should be magnified for the slower process. Thus, if there is a difference between category and property retrieval times, we should expect the difference to interact with $\mathrm{PF}$, such that there is a greater difference for low-PF predicates. Featural theories would not make this prediction. A significant difference that did not interact with PF would therefore not be readily explainable in terms of search-andretrieval mechanisms.

The logic of this argument is directly comparable to that of Sternberg's (1969) methodology. A major issue (Smith, 1978) between network and featural models is the relative importance of the retrieval of prestored information (as in network-search models) and the computation of new decisions (as in feature-comparison models). If there is any difference in verification time for category and property information, then it may be possible to use Sternberg's methodology to assess whether that difference is occurring at a retrieval stage or at some other stage. Sternberg argued that, when two independent variables affect the same stage of an informationprocessing sequence, then their effects on overall time will interact (generally speaking), whereas if they affect separate stages, their effects will be additive. [Recent treatments of this method (McClelland, 1979; Schweickert, 1983) have concurred that additivity is a sign of separate stages, although an interaction need not always imply that the variables are affecting the same stage.] Now, since associative PF is a direct measure of the retrievability of the information, we may assume that retrieval-based models should predict an interaction between predicate type and PF. On the other hand, discovery of a predicate-type effect that was independent of PF would argue against any retrieval-based explanation of the predicate-type difference, and consequently argue against the central importance of retrieval processes in determining verification time.
The aim of the experiments, therefore, was, first, to determine which (if either) type of predicate was more rapidly verified and, second, to discover whether any difference between predicate types interacted with PF. The experiments aimed to provide an answer to an empirical question, the answer to which should constrain the development of models of semantic memory. Although neither approach to modeling semantic memory provides a clear prediction of a difference between predicate types, the discovery of such a difference could nevertheless be used to partially discriminate between classes of models.

\section{EXPERIMENT 1}

\section{Method}

Subjects. Twenty-four student volunteers (10 males and 14 females) at The City University, London, acted as paid subjects; all were native speakers of English and were right-handed.

Design. A two-factor repeated-measure design was employed, the factors were predicate type (category vs. property) and truth (true vs. false). Nested within the true sentences, there was an additional factor of PF (high, medium, or low).

Materials. Four predicates were selected for each of 64 subject nouns. These predicates were two category statements (one true and one false) and two property statements (one true and one false). True predicates were selected for each subject noun so that category and property statements were matched for $\mathrm{PF}$ within one of three levels-high ( $\mathrm{PF}=33 \%$ to $94 \%$ ), medium $(\mathrm{PF}=15 \%$ to $33 \%)$, and low $(\mathrm{PF}=4 \%$ to $15 \%)$. There were approximately the same number of subject nouns for each level of PF. The predicates themselves were chosen from three sets of property norms [Ashcraft, 1976a, 1976b (also published in shorter form-Ashcraft, 1978b) and two additional sets of unpublished data collected by the author (based on 24 and 16 subjects, respectively)]. PF was balanced for category and property statements within each set of norms. [The subjects generating these norms simply listed any information that came to mind to describe and define the concepts named by each noun, with no constraint being placed on the type of information produced. Production was not time constrained in the author's data, but Ashcraft (1978b) allowed $40 \mathrm{sec}$ per word. Subjects typically produced between 4 and 10 different predicates to each noun. Production order of predicates was not controlled, but has been shown to correlate well with PF (Hampton, 1976). Only 3 predicates that had PFs greater than $80 \%$ were used, so differences in associative strength were not concealed by ceiling effects.] The lengths of the two predicates were also matched. Each predicate (category or property) occurred only once as a true sentence and once as a false sentence. Each subject noun occurred four times, once under each truth $x$ predicate type condition. False sentences were constructed by reassigning predicates to subject nouns to produce unambiguously false statements. The resulting 256 sentences were divided randomly into four blocks so that each subject noun occurred once in each block, no predicate occurred twice in the same block, and there were equal numbers of the four sentence types in each block. Each subject received a different random sequence of the sentences in each block, and the order of blocks was balanced across subjects. A list of the sentences used is given in the Appendix.

Apparatus. A Commodore 3016 microcomputer was programmed to display the sentences one at a time on its VDU screen and to record the subject's responses and reaction times in milliseconds. The subject was seated in front of the display screen and had two response keys to press, one by each of his or her hands. 
Procedure. A warning signal (an asterisk) appeared in the center of the screen for $1.5 \mathrm{sec}$ before the display of the subject noun above the predicate, both in uppercase characters. These were displayed until a key was pressed. There was then a 3 -sec blank interval before the warning signal appeared for the next trial. If the noun plus predicate made a sentence that was "generally true," the subject was to press the right-hand key, and if false, the left-hand key. The subject was told that all the sentences were obviously true or false. If the subject thought a sentence meaningless, he or she was to respond "false." The subject was instructed to respond as fast as possible while mak ing as few errors as he or she could. The first 10 trials were practice materials and were not recorded. Between each of the four blocks of 64 trials, the subject was allowed to rest for as long as he or she wished; the subject started the next block by pressing either response key. Experiment 1 lasted about $25 \mathrm{~min}$.

\section{Results}

Before mean response times were calculated, erroneous responses ( $6 \%$ of the data) were removed. In addition, 26 latencies of over $3 \mathrm{sec}$ and five machine errors were removed from the data. The mean and standard deviation for correct reaction times for each condition are shown in Table 1, together with error rates. For both true and false statements, category predicates were responded to faster than property predicates. The differences were $121 \mathrm{msec}$ for true and $110 \mathrm{msec}$ for false predicates.

A two-way analysis of variance with repeated-measures factors of truth and predicate type showed a significant main effect of predicate type $\left[\min F^{\prime}(1,50)=36.2\right.$, $\mathrm{p}<.001]$. There was no main effect of truth and no interaction ( $F<1$ in both cases). Across materials, the size of the category advantage did not correlate with mean PF $(r=.08, n=64)$. An analysis of error rates gave significant factors of truth $\left[\min \mathrm{F}^{\prime}(1,54)=10.47\right.$, $\mathrm{p}<.01]$ and predicate type $\left[\min \mathrm{F}^{\prime}(1,40)=4.4, \mathrm{p}<\right.$ $.05]$, but no significant interaction $\left(\min F^{\prime}<1\right)$. True sentences had more errors than false, and property predicates had more errors than category predicates. (A closer analysis of the error distribution revealed that certain sentences had significantly higher error rates than would be expected from a random distribution of errors across materials. There were eight category and eight property statements involved. Reanalysis of the reaction time and error rate data excluding these sentences showed no change in the size or significance of the effects reported above.)
Finally, mean response times for true sentences were analyzed to discover whether the observed significant factor of predicate type interacted with the PF of the predicates. Mean reaction times for the three levels of PF for each predicate type are also shown in Table 1, in which it can be seen that the category advantage was present at all three levels. Analysis of variance across materials confirmed the main effect of predicate type $[F(1,61)=27.3, p<.001]$. There was a slight attenuation of the effect at low PF, but the interaction did not approach significance $[F(2,61)<1]$. The main effect of $\mathrm{PF}$ itself was significant $[\mathrm{F}(2,61)=7.21, \mathrm{p}<.01]$. As would be expected, high-PF sentences $(1,037 \mathrm{msec})$ were faster than medium-PF sentences $(1,110 \mathrm{msec})$, which were faster than low-PF sentences $(1,165 \mathrm{msec})$. A corresponding analysis of false sentences, treating $\mathrm{PF}$ of the predicate as a pseudovariable, showed no increase in verification speed as $\mathrm{PF}$ dropped (mean times were: high PF-1,106 msec, medium PF-1,083 msec, low PF$1,093 \mathrm{msec})$. The PF effect was therefore due to the relation between subject noun and predicate, and not to the predicate alone.

\section{Discussion}

The experiment provided clear results. Whether true or false, the category statements were responded to over $100 \mathrm{msec}$ faster than the property statements. There was a difference in the error rate in the same direction (therefore ruling out any explanation in terms of speedaccuracy tradeoffs). There was also a tendency to make more false-negative than false-positive errors. Most importantly for discriminating between semantic memory models, the category advantage did not interact with PF. Indeed it appeared to be less strong with lower PF.

The existence of equivalent-sized effects for true and false sentences (interaction $F<1$ ) suggests that the result for true sentences cannot be attributed to any hidden imbalance in associative strength of category and property predicates, since such an imbalance would not affect the false sentences, except indirectly.

Before any strong conclusions were drawn from this result, it was deemed advisable to replicate the experiment to ensure that the result was not an artifact of the materials used. Specifically, there was an obvious im-

Table 1

Means and Standard Deviations for Reaction Times (RTs, in Milliseconds) and Error Rates (in Percent) for Sentences in Experiment 1

\begin{tabular}{|c|c|c|c|c|c|c|c|c|}
\hline \multirow[b]{2}{*}{ PF } & \multicolumn{3}{|c|}{ Category } & \multicolumn{3}{|c|}{ Property } & \multirow{2}{*}{$\begin{array}{c}\text { Number of } \\
\text { Words }\end{array}$} & \multirow{2}{*}{$\begin{array}{l}\text { Category } \\
\text { Advantage }\end{array}$} \\
\hline & Mean RT & $\mathrm{SD}$ & ER & Mean RT & SD & ER & & \\
\hline \multicolumn{9}{|c|}{ True Sentences } \\
\hline High & 967 & 125 & 3 & 1107 & 141 & 7 & 20 & 140 \\
\hline Medium & 1040 & 112 & 10 & 1181 & 170 & 7 & 16 & 141 \\
\hline Low & 1119 & 155 & 11 & 1211 & 163 & 11 & 28 & 92 \\
\hline Total & 1048 & 189 & 8 & 1169 & 222 & 9 & 64 & 121 \\
\hline \multicolumn{9}{|c|}{ False Sentences } \\
\hline & 1043 & 181 & 2 & 1153 & 228 & 6 & 64 & 110 \\
\hline
\end{tabular}


balance in the two types of predicate in terms of the variety of syntactic form used. Category predicates had the standard form "is (a) [NOUN]," whereas property predicates could be of three types- "is [ADJECTIVE]," "has a [NOUN]," or one containing some specific verb (e.g., "grows in summer"). Although post hoc analysis showed the category advantage to be strongest for the words with the "is [ADJECTIVE]" type of property, it was felt that the subjects may have been sensitive to the different frequencies of syntactic forms. There were also more adjective-noun combinations in the property predicates. Experiment 2, therefore, used only one form of property predicate, namely, "has a [NOUN]." Using this form also enabled the word frequency of the predicate noun to be controlled.

\section{EXPERIMENT 2}

\section{Method}

Subjects. Twenty-four student volunteers at The City University, London, acted as paid subjects. There were roughly equal numbers of males and females, and they were all righthanded native speakers of English.

Design and Materials. The same design was used that had been used in Experiment 1, with the two factors of truth and predicate type, and with PF nested within true sentences. The sentences were constructed as follows. Fifty-two subject nouns were used (45 of which were used in Experiment 1), and a category and a property predicate were selected for each word from the same norms used previously, so that associative PF, length, and word frequency (Kucera \& Francis, 1967) were all matched. PF was matched at three levels, as in Experiment 1. All the property predicates contained the auxiliary verb "has," and all category predicates had the verb "is." In addition, no predicates were used that had given rise to a significant number of errors in Experiment 1 . As before, each predicate occurred once only as a true sentence. False sentences were created in the same way as before. Three judges read through the false sentences, picking out any that contained related concepts. On average, only 6 of the 104 were selected as related, and these were evenly distributed between category and property statements. Since agreement between judges was poor, no items were rejected. Randomization and balancing followed the same design as Experiment 1, with four blocks of 52 trials, presented in random sequence. The materials used are shown in the Appendix.

Procedure and Apparatus. The details of procedure and apparatus were identical to those for Experiment 1, with the exception that slightly fewer sentences were used, so that the duration of the experiment was approximately $20 \mathrm{~min}$.

\section{Results}

Error response times and 38 latencies of over $3 \mathrm{sec}$ were removed from the data. The average error rate was $6 \%$. Table 2 shows the mean correct reaction times with standard deviations and error rates for the four types of sentence. Category predicates were again responded to faster than property predicates. The difference was $73 \mathrm{msec}$ for true and $53 \mathrm{msec}$ for false sentences. A two-way repeated-measures analysis of variance confirmed the main effect of predicate type $\left[\min F^{\prime}(1,40)=12.48, p<.01\right]$, with no effect of truth and no interaction ( $F<1$ in both cases). Error rates were also subjected to analysis of variance. There was a significant main effect of truth $\left[\min \mathrm{F}^{\prime}(1,39)=\right.$ $16.72, \mathrm{p}<.001]$, but no significant effect of predicate type $\left[\min F^{\prime}(1,41)=1.83, p>.20\right]$, unlike in Experiment 1 . There was no interaction $(F<1)$. As before, true sentences were more prone to errors than false ones.

Analysis of the response times for true sentences with respect to predicate type and PF revealed a pattern similar to that in Experiment 1. There were significant main effects of predicate type $\left[\min F^{\prime}(1,34)=6.85\right.$, $\mathrm{p}<.05]$ and of $\mathrm{PF}\left[\min \mathrm{F}^{\prime}(2,56)=4.6, \mathrm{p}<.05\right]$, but no significant interaction [across subjects, $F(2,46)=$ 2.16 ; across materials, $F(2,49)=0.14]$. The category advantage was strongest at medium PF $(103 \mathrm{msec})$, and less strong at high or at low PF (71 and $45 \mathrm{msec}$, respectively). The means are shown in Table 2 . The size of the category advantage did not correlate at all with $\mathrm{PF}(\mathrm{r}=-0.05, \mathrm{n}=52)$.

As found in Experiment 1, mean decision times for false sentences were equivalent across PF levels (high PF-1,085 msec, medium $\mathrm{PF}-1,087 \mathrm{msec}$, and low $\mathrm{PF}-1,089 \mathrm{msec}$ ), so that the PF effect for true sentences was due to the relation between subject and predicate, and not to the predicate alone.

As an additional control for confounding variables associated with predicate retrieval, a condition was run in which 20 new subjects from the same population read

Table 2

Means and Standard Deviations for Reaction Times (RTs, in Milliseconds) and Error Rates (in Percent) for Sentences in Experiment 2

\begin{tabular}{|c|c|c|c|c|c|c|c|c|}
\hline \multirow[b]{2}{*}{ PF } & \multicolumn{3}{|c|}{ Category } & \multicolumn{3}{|c|}{ Property } & \multirow{2}{*}{$\begin{array}{c}\text { Number of } \\
\text { Words }\end{array}$} & \multirow{2}{*}{$\begin{array}{l}\text { Category } \\
\text { Advantage }\end{array}$} \\
\hline & Mean RT & SD & $\overline{E R}$ & Mean RT & SD & ER & & \\
\hline \multicolumn{9}{|c|}{ True Sentences } \\
\hline $\begin{array}{l}\text { High } \\
\text { Medium } \\
\text { Low }\end{array}$ & $\begin{array}{l}1010 \\
1005 \\
1135\end{array}$ & $\begin{array}{l}211 \\
218 \\
260\end{array}$ & $\begin{array}{r}10 \\
4 \\
11\end{array}$ & $\begin{array}{l}1081 \\
1108 \\
1180\end{array}$ & $\begin{array}{l}261 \\
252 \\
286\end{array}$ & $\begin{array}{r}9 \\
8 \\
14\end{array}$ & $\begin{array}{l}20 \\
15 \\
17\end{array}$ & $\begin{array}{r}71 \\
103 \\
45\end{array}$ \\
\hline Total & 1050 & 224 & 8 & 1123 & 262 & 11 & 52 & 73 \\
\hline \multicolumn{9}{|c|}{ False Sentences } \\
\hline & 1062 & 210 & 3 & 1115 & 226 & 4 & 52 & 53 \\
\hline
\end{tabular}


through the full list of true and false sentences. Sentence presentation began with display of the subject noun. When the subject pressed a key with his or her left hand, the predicate was then displayed. The subject pressed a second key with the right hand as soon as he or she had read and understood the predicate. Thus, no decision was required; the subject merely had to read the sentence subject and predicate. The two inspection times were recorded separately, and time to read the predicate was analyzed across materials in a $2 \times 2 \times 3$ analysis of variance with factors of truth, predicate type, and $\mathrm{PF}$ (as a true sentence). There was a significant effect of truth $[\mathrm{F}(1,49)=17.2, \mathrm{MSe}=2,575, \mathrm{p}<.0001]$, with true predicates being read faster $(583 \mathrm{msec})$ than false $(612 \mathrm{msec})$, but no other main effects or interactions were significant. Mean reading times are shown in Table 3. Overall, category predicates $(598 \mathrm{msec})$ were read no faster than property predicates $(598 \mathrm{msec})$. The power of the test for this comparison was .75 for obtaining a difference of $45 \mathrm{msec}$ or more, and .99 for a difference of as much as $75 \mathrm{msec}$. It can therefore be safely concluded that observed differences in verification time were not attributable to differences in reading time for the predicates, even in the context of their subject nouns. The significant effect of truth confirmed that subjects were in fact reading the sentences for their meaning. There was a small effect of PF on an analysis of the true sentences only; the effect was significant across subjects but not across materials. (Low-PF sentences were read, on average, $26 \mathrm{msec}$ slower than medium- or high-PF sentences.) The reading control condition therefore supported the major conclusions of Experiment 2.

A final question concerns the absence of an interaction between predicate type and PF for the true sentences. The interpretation of this null effect must be treated with some caution in the light of the low power of the test. The error variance across materials was such that several hundred sentences would be required to provide an adequate demonstration of additivity in a single experiment. In order to examine the possible interaction more carefully, an analysis of covariance was conducted across materials for the true sentences,

Table 3

Means and Standard Deviations for Reading Times (in Milliseconds) for the Control Condition in Experiment 2

\begin{tabular}{|c|c|c|c|c|}
\hline \multirow[b]{2}{*}{ PF } & \multicolumn{2}{|c|}{ Category } & \multicolumn{2}{|c|}{ Property } \\
\hline & $\begin{array}{c}\text { Mean } \\
\text { Reading Time }\end{array}$ & SD & $\begin{array}{c}\text { Mean } \\
\text { Reading Time }\end{array}$ & $\mathrm{SD}$ \\
\hline \multicolumn{5}{|c|}{ True Sentences } \\
\hline $\begin{array}{l}\text { High } \\
\text { Medium } \\
\text { Low }\end{array}$ & $\begin{array}{l}578 \\
581 \\
599\end{array}$ & $\begin{array}{l}61 \\
39 \\
44\end{array}$ & $\begin{array}{l}574 \\
567 \\
603\end{array}$ & $\begin{array}{l}44 \\
40 \\
49\end{array}$ \\
\hline Total & 586 & 50 & 581 & 46 \\
\hline \multicolumn{5}{|c|}{ False Sentences } \\
\hline & 609 & 56 & 615 & 44 \\
\hline
\end{tabular}

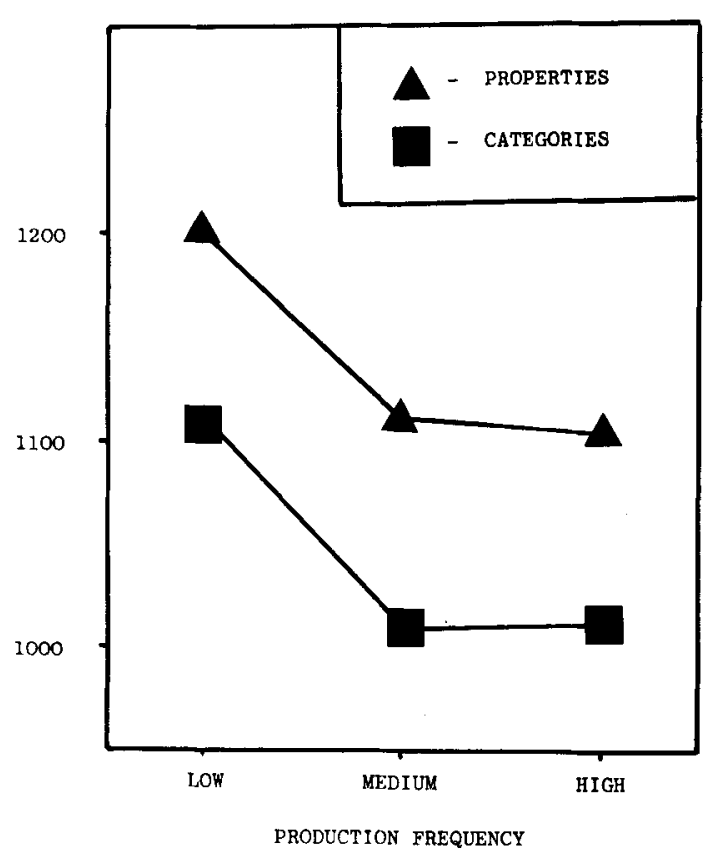

Figure 1. Corrected mean reaction times for Experiment 2, controlling for the effects of four covariates: PF, length, reading time, and word frequency.

with factors of PF and predicate type, and with covariates for each predicate (nested within predicate type) of PF, length, word frequency, and reading time. Removing these extraneous sources of variance should have the effect of rendering a clearer picture of any underlying interaction between PF and predicate type. The results showed again a strong effect of predicate type $[\mathrm{F}(1,45)=11.58, \mathrm{MSe}=18,516, \mathrm{p}<.002]$ but no hint of an interaction $[F(2,45)=0.04$, MSe $=18,516$, $p=.96]$. As can be seen in Figure 1, the slightly greater category advantage obtained for medium-PF sentences was attenuated by the control of the covariates, and the data appear to demonstrate additivity. (There was a residual effect of the factor of $P F$, which was due to a nonlinear relation between verification time and PF level as defined in the present sample.) It may also be noted that the test for a correlation between category advantage and mean PF across predicate pairs reported previously had a power of .75 to reveal an association of .32 or greater $(\mathrm{r}=-0.05)$. Although the interaction test had low power, the absence of any sign of an increase in the advantage to category predicates with lower PF can be taken as a failure to support a retrieval-based account of the effect. This issue is discussed further in the final section.

\section{Discussion}

Experiment 2 replicated the results of Experiment 1 in almost every respect. In addition, the stimulus frequency of sentence forms and the word frequency of predicate nouns were controlled, and a measure of reading time showed no effect of predicate type. ${ }^{1}$ The relative advantage of category predicates in both true and 
false sentences was found in the response times but, unlike Experiment 1, did not reach significance in the analysis of error rates. The bias toward false-negative rather than false-positive errors was also replicated.

It may be concluded from the successful replication that the category advantage obtained in Experiment 1 was not attributable to uncontrolled stimulus-frequency effects. In both experiments, there was a reliable advantage for the verification of category information that did not interact with PF. The following section discusses the implications of this result.

\section{GENERAL DISCUSSION}

A consistent pattern of results was obtained from these two experiments, the first of which used a broad range of properties, and the second a more constrained set. The results showed category information to be verified more quickly than property information of the same associative and word frequency. This advantage was found for both true and false sentences, and for true sentences it was apparently independent of PF. No current model of semantic memory would readily predict this result. How then may it best be interpreted?

Essentially, all models of semantic memory recognize that information must first be retrieved and then decided upon, but, as Smith (1978) argued, a critical difference between models lies in the amount of work and time assumed to be involved in either retrieval or decision processes. Most network models (e.g., Glass \& Holyoak, 1975) emphasize the role of retrieval in verification. If retrieval is the key factor, then, when PF is equated, one would not expect there to be any substantial residual reliable difference in verification times. Indeed, Conrad (1972) showed that, for a property verification task, with $P F$ held constant, there was no residual effect of number of links traversed in the network on response time. For the data reported here, a post hoc division of the true property sentences into those specific to the subject noun (e.g., "Croquet has hoops") and those that might be inferred from membership of some higher level category (e.g., "Owl has feathers") likewise showed no difference in the size of the category advantage for the two types of propertyin either experiment. This result confirms Conrad's conclusion that Collins and Quillian's (1969) principle of cognitive economy is unsupported. It could certainly not explain the observed differences in verification times. Although often cited as a failure of network theory, it could in fact be argued that Conrad's result is exactly what would be expected if verification depends largely on retrieval (since distance in the network should determine both retrieval time and PF). A similar result reported by Smith et al. (1974), in which categorization was faster in a more distant category ("A chicken is an animal") than in a closer category ("A chicken is a bird"), could also be accounted for by the general principle of associative frequency, operating in a retrieval model (e.g., Glass \& Holyoak, 1975). "Animal" is a more frequently produced superordinate to "chicken" than is "bird." Hence, Marbe's law still predicts the result.

The present result is therefore a more serious finding for retrieval models in general than these often quoted critiques. Unlike Conrad's (1972) and Smith et al.'s (1974) results, Marbe's law fails to account for the observed difference between category and property information verification. Furthermore, it would be hard to modify a retrieval model to account for the difference, given that the result occurs equally at high and at low PF, and as strongly for true as for false sentences.

The lack of interaction between the category ad. vantage and $\mathrm{PF}$ in these two experiments was confirmed in an independent study (Hampton \& Port, 1983) recently conducted in our laboratory. Thirty-two words were divided into two groups matched for familiarity, and a group of subjects each generated several category and "has"-property predicates to each word in both groups. Matched pairs of predicates (one category and one property) were selected at high and low PF from this task (one group of words had high-PF predicates, and the other had low-PF predicates). A second group of 32 subjects then performed a timed verification task on these sentences. The advantage to category predicates was replicated $(72 \mathrm{msec}$ for true and $86 \mathrm{msec}$ for false), and the advantage was slightly lower at low PF ( 80 msec for high PF and $63 \mathrm{msec}$ for low PF). There were significant main effects of predicate type and PF, but no significant interaction [across materials, $F(1,28)=0.08 ;$ across subjects, $F(1,31)=0.66]$. There are therefore now three studies in which the category advantage has been replicated, and in no case was there any sign of an increase in the effect at low PF. Taken together, this repeated failure to find the predicted interaction suggests that the retrieval-based account is inadequate. Following Sternberg's (1969) logic, it appears likely that PF effects and the bases of the category advantage are located at different stages in the verification process.

Given that the difference is unlikely to lie in the retrieval of prestored information, the alternative locus for the effect would be in a decision stage. Featurecomparison models of categorization (Hampton, 1979; McCloskey \& Glucksberg, 1979; Smith et al., 1974) concentrate on the decision stage as the source of observed differences in verification time. In support of locating the category advantage at a decision stage, there is the fact that the advantage is independent of PF and occurs equally strongly for true and for false sentences. ${ }^{2}$ Possible accounts of the effect might then be framed along the following lines.

It is recognized, largely from the work of Rosch and her colleagues (Rosch \& Mervis, 1975), that categories are formed to maximize the family resemblances of items within a single category and to minimize the similarity between items that fall in different categories. 
For any category decision, there are therefore a number of correlated features or dimensions that can be used for making the judgment, and because of their positive correlation, the probability is high that most items in a domain will fall clearly in or clearly out of a particular category. This is because the features constituting the prototype representation of a category will be weighted in order to maximize the cohesiveness of the group of category members. There are, of course, notable exceptions to the clear-cut boundaries of categories (see Hampton, 1979, 1982, and McCloskey \& Glucksberg, 1978). However, their number is necessarily small compared with the large cluster of items that are clear examples.

If we consider property predicates, there is much less evidence for any such structure. Usually, some single specific physical or functional piece of information about the concept must be retrieved and used. There will be few correlated features that could make the decision easier, and thus true sentences will be harder to verify. A first explanation of the category advantage therefore lies in the degree of specificity and lack of redundancy in the information needed to verify properties as opposed to category statements.

A second account concerns the ease with which sentences can be rejected as false, through the discovery of directly contradicting information. Category information is frequently (although not always) taxonomic in structure. That is to say, a domain (such as creatures) will be divided up into mutually exclusive sets of items (such as fish, birds, insects, and so on). As represented psychologically, such knowledge is frequently incomplete and less exact than is sometimes supposed (for examples, see Hampton, 1982), but nonetheless there are still many examples of mutually exclusive sets. $\mathrm{Ob}$ viously, if an item belongs in such a set, then this fact can be used to infer rapidly that it is not in any other of the sets in the domain. (For evidence of this contradiction strategy, see Anderson \& Reder, 1974, and Holyoak \& Glass, 1975.) For property statements, however, there appears again to be less structure. Properties do not form mutually exclusive subdivisions of a domain to the same extent. Overlap between the classes defined by possession of a particular property is usually high (consider, e.g., the sets of creatures having tails, wings, legs, or eyes). Thus, the contradiction strategy that can be used for some category judgments is unlikely to be of use in falsifying properties.

These accounts must of course be speculative, and further study of property verification is clearly called for. It would, for example, be interesting to compare category predicates with false property predicates that either did or did not directly contradict a true property. Recently, studies (Ashcraft, 1978a; Barsalou, 1982) have indicated some of the variety of properties involved in concept representations. Whatever the fate of the suggestions made here, the main significance of the present experiments lies in the demonstration of a strong and reliable effect on sentence verification time that cannot be explained in terms of Marbe's law of associations. Future developments in semantic memory must consider how the decision processes involved in verifying category and property information operate, in order to produce this difference in their ease of verification.

\section{REFERENCES}

Anderson, J. R., \& Reder, L. M. (1974). Negative judgments in and about semantic memory. Journal of Verbal Learning and Verbal Behavior, 13, 664-681.

Ashcraft, M. H. (1976a). Priming and property dominance effects in semantic memory. Memory \& Cognition, 4, 490-500.

Ashcraft, M. H. (1976b). Property norms for typical and atypical items from 17 categories. Unpublished manuscript, Cleveland State University.

Ashcraft, M. H. (1978a). Property dominance and typicality effects in property statement verification. Journal of Verbal Learning and Verbal Behavior, 17, 155-164.

Ashcraft, M. H. (1978b). Property norms for typical and atypical items from 17 categories: A description and discussion. Memory \& Cognition, 6, 227-232.

Barsalou, L. W. (1982). Context-independent and contextdependent information in concepts. Memory \& Cognition, 10, 82-93.

Collins, A. M., \& Loftus, E. F. (1975). A spreading-activation theory of semantic processing. Psychological Review, 82, 407-428.

Collins, A. M., \& Quillian, M. R. (1969). Retrieval time from semantic memory. Journal of Verbal Learning and Verbal Behavior, 8, 240-248.

Collins, A. M., \& Quillian, M. R. (1972). How to make a language user. In E. Tulving \& W. Donaldson (Eds.), Organization of Memory. New York: Academic Press.

Conrad, C. E. H. (1972). Cognitive economy in semantic memory. Journal of Experimental Psychology, 92, 149-154.

Glass, A. L., \& HolyoAK, K. J..(1975). Alternative conceptions of semantic memory. Cognition, 3, 313-339.

Glass, A. L., Holyoak, K. J., \& O'Dell, C. (1974). Production frequency and the verification of quantified statements. Journal of Verbal Learning and Verbal Behavior, 13, 237-254.

HAM PTON, J. A. (1976). An experimental study of concepts in language. Unpublished doctoral thesis, University of London.

Hampton, J. A. (1979). Polymorphous concepts in semantic memory. Journal of Verbal Learning and Verbal Behavior, 18, 441-461.

Hampton, J. A. (1981). An investigation of the nature of abstract concepts. Memory \& Cognition, 9, 149-156.

HAmpton, J. A. (1982). A demonstration of intransitivity in natural categories. Cognition, 12, 151-164.

Hampton, J. A., \& PoRT, J. (1983). Effects of production frequency, predicate type and subject noun familiarity on sentence verification time. Unpublished manuscript, The City University, London.

Holyoak, K. J., \& Glass, A. L. (1975). The role of contradictions and counterexamples in the rejection of false sentences. Journal of Verbal Learning and Verbal Behavior, 14, 215-239.

Johnson-Laird, P. N. (1975). Meaning and the mental lexicon. In A. Kennedy \& A. Wilkes (Eds.), Studies in long term memory. London: Wiley.

Kučera, H., : Francis, W. N. (1967). Computational analysis of present-day English. Providence, RI: Brown University Press.

LoFrus, E. F. (1973). Category dominance, instance dominance and categorization time. Journal of Experimental Psychology, 97, 70-74.

MCClelland, J. L. (1979). On the time relations of mental pro- 
cesses: An examination of systems of processes in cascade. Psychological Review, 86, 287-330.

McCloskey, M., \& Glucksberg, S. (1978). Natural categories: Well defined or fuzzy sets? Memory \& Cognition, 6, 462-472.

McCloskey, M., \& Glucksberg, S. (1979). Decision processes in verifying category membership statements: Implications for models of semantic memory. Cognitive Psychology, 11, 1-37.

Rosch, E., \& Mervis, C. B. (1975). Family resemblances: Studies in the internal structure of categories. Cognitive Psychology, 7, 573-605.

Schaeffer, B., \& Wallace, R. (1970). The comparison of word meanings. Journal of Experimental Psychology, 86, 144-152.

ScHWEICKERT, R. (1983). Latent network theory: Scheduling of processes in sentence verification and the Stroop effect. Journal of Experimental Psychology: Learning, Memory, and Cognition, 9, 353-383.

Smith, E. E. (1978). Theories of semantic memory. In W. K. Estes (Ed.), Handbook of learning and cognitive processes (Vol. 5). Hillsdale, NJ: Erlbaum.

Smith, E. E., Shoben, E. J., \& RiPs, L. J. (1974). Structure and process in semantic memory: A featural model for semantic decisions. Psychological Review, 81, 214-241.

STERnBerg, S. (1969). The discovery of processing stages: Ex- tensions of Donder's method. Acta Psychologica, 30, 276-315.

WierzbickA, A. (1972). Semantic primitives (A. Wierzbicka \& J. Besemeres, Trans.). Frankfurt: Athenaum.

Wilkins, A. T. (1971). Conjoint frequency, category size and categorization time. Journal of Verbal Learning and Verbal Behavior, 10, 382-385.

\section{NOTES}

1. It should be noted that although syntactic form was controlled, there may still be a confounding effect of semantic variety, in that the "has" sentences included a wider range of semantic relations (such as part-whole vs. alienable possession).

2 . The nearly equal size of the predicate-type effect for true and false sentences (121 vs. $110 \mathrm{msec}$, and 73 vs. $53 \mathrm{msec}$ in each experiment) need not be taken to imply that predicatetype differences affect a stage distinct from the stage in which the correct response is selected. Given that predicate type affects the ease of discriminating true from false sentences (for whatever reason), one would expect such an effect to appear in the response times for both true and false responses-as indeed was the case in the present experiments.

APPENDIX

Materials Used in the Experiments

\begin{tabular}{|c|c|c|c|c|}
\hline Word & True Category & True Property & False Category & False Property \\
\hline \multicolumn{5}{|c|}{ Experiment 1} \\
\hline \multicolumn{5}{|l|}{ High PF } \\
\hline Oak & Is a tree & Has green leaves & Is a creature & Eats mice \\
\hline Sparrow & Is a bird & Has wings & Is a tree & Is used for stockings \\
\hline Rose & Is a flower & Has sharp thorns & Is a mammal & Has wings \\
\hline Grape & Is a fruit & Contains small seeds & Is a building & Is used for sheets \\
\hline Violin & Is a musical instrument & Is made of wood & Is a grain & Lives in forests \\
\hline Tea & Is a drink & Is made from leaves & Is a construction & Is often dirty \\
\hline Ant & Is an insect & Is black & Is a flower & Is made from leaves \\
\hline Hammer & Is a tool & Is metal & Is a bug & Contains small seeds \\
\hline Linen & Is a cloth & Is used for sheets & Is a beverage & Has a mouth \\
\hline Hotel & Is a building & Has many beds & Is a musical instrument & Has pockets \\
\hline Garlic & Is a food flavouring & Is used in cooking & Is a hobby & Is strongly built \\
\hline Octopus & Is a creature & Has long tentacles & Is poultry & Is for carrying goods \\
\hline Van & Is transport & Is for carrying goods & Is a delicacy & Uses sharp hooks \\
\hline Football & Is a sport & Has two teams & Is a plant & Is used in cooking \\
\hline Croquet & Is a game & Uses wooden mallets & Is a citrus fruit & Has small white pips \\
\hline Onion & Is a vegetable & Makes you cry & Is a primate & Stands against a wall \\
\hline Eel & Is a fish & Is very slimy & Is an act & Is vertical \\
\hline Potato & Is a staple food & Has a skin & Is a rodent & Makes you cry \\
\hline Ship & Is a vessel & Floats on water & Is a staple food & Is found in parks \\
\hline Lemon & Is a citrus fruit & Has small white pips & Is a fowl & Uses wooden mallets \\
\hline \multicolumn{5}{|l|}{ Medium PF } \\
\hline Sword & Is a weapon & Has a blade & Is a fruit & Is drunk with cream \\
\hline Cabin & Is a house & Has a window & Is food & Is very crisp \\
\hline Rice & Is a grain & Contains starch & Is transport & Opens up boxes \\
\hline Pine & Is a wood & Has a smell & Is a game & Has sharp thorns \\
\hline Sofa & Is furniture & Is for sitting on & Is a parasite & Is hard to control \\
\hline Nylon & Is a synthetic fibre & Is used for stockings & Is seafood & Eats through wool \\
\hline Coffee & Is a beverage & Is drunk with cream & Is a tool & Is used for sails \\
\hline Celery & Is a plant & Is very crisp & Is a predator & Has pink legs \\
\hline Trout & Is food & Has a mouth & Is a wood & Has green leaves \\
\hline Angling & Is a hobby & Uses sharp hooks & Is an object & Is battery bred \\
\hline Rug & Is a floor covering & Is for comfort & Is a sport & Has a skin \\
\hline Arrow & Is a projectile & Has feathered flights & Is a food-flavouring & Is very slimy \\
\hline Coughing & Is an act & Is hard to control & Is a kitchen object & Floats on water \\
\hline Lamb & Is a kind of meat & Has four legs & Is a device & Has a blade \\
\hline Ladder & Is a device & Is strongly built & Is a vessel & Is for comfort \\
\hline Sunbathing & Is an activity & Can cause sunburn & Is a container & Is kept sharp \\
\hline \multicolumn{5}{|l|}{ Low PF } \\
\hline Coat & Is clothing & Has pockets & Is a vehicle & Is driven \\
\hline Shrimp & Is seafood & Has pink legs & Is furniture & Is for sitting on \\
\hline
\end{tabular}


Word

Flea

Hut

Moth

Cotton

Crowbar

Car

Drum

Parsnip

Bear

Robin

Daisy

Owl

Canvas

Lion

Salmon

Raft

Chicken

Chair

Otter

Bookcase

Man

Knife

Duck

Corkscrew

Door

Pavement

High PF

Oak

Celery

Owl

Grape

Spider

Hammer

Hotel

Garlic

Octopus

Van

Croquet

Daisy

Dustbin

Eel

Otter

Rose

Arrow

Onion

Lemon

Ship

Medium PF

Drum

Sword

Cabin

Rice

Shrimp

Sofa

Coat

Trout

Bamboo

Truck

Football

Chicken

Angling

Man

Fence

Low PF

Hut

Moth

Crowbar

Car
True Category

Is a parasite

Is a shelter

Is a bug

Is a material

Is a lever

Is a vehicle

Is a cylinder

Is a root crop

Is a mammal

Is an animal

Is a weed

Is a predator

Is a woven fabric

Is a carnivore

Is a delicacy

Is a primitive craft

Is poultry

Is an object

Is a rodent

Is a container

Is a primate

Is cutlery

Is a fowl

Is a kitchen object

Is a construction

Is a path

Is a tree

Is a vegetable

Is a bird

Is a fruit

Is an insect

Is a tool

Is a building

Is a flavouring

Is a creature

Is transport

Is a game

Is a flower

Is a cylinder

Is a fish

Is an animal

Is a bush

Is a projectile

Is a tuber

Is a citrus fruit

Is a vessel

Is an instrument

Is a weapon

Is a house

Is a grain

Is seafood

Is furniture

Is clothing

Is food

Is a plant

Is a vehicle

Is a sport

Is a fowl

Is a pastime

Is a primate

Is a barrier

Is a shelter

Is a bug

Is a lever

Is an automobile

True Property

False Category

False Property

Sucks animal's blood

Has a roof

Eats through wood

Is made into clothes

Opens up boxes

Is driven

Is hollow inside

Is nutritious

Lives in forests

Has a tail

Grows in summer

Eats mice

Is used for sails

Is very powerful

Is eaten smoked

Is square

Is battery bred

Is for individuals

Has whiskers

Stands against a wall

Has two hands

Is kept sharp

Is found in parks

Is useful

Is vertical

Is often dirty

Experiment 2

Has leaves

Has stalks

Has feathers

Has seeds

Has a web

Has a head

Has beds

Has a smell

Has tentacles

Has an engine

Has hoops

Has petals

Has a lid

Has slime

Has fur

Has a scent

Has flights

Has layers

Has white pips

Has a funnel

Has a skin

Has a point

Has a window

Has starch

Has a tail

Has springs

Has a collar

Has a mouth

Has shoots

Has a driver

Has a pitch

Has a beak

Has competitions

Has a brain

Has posts

Has a roof

Has legs

Has a bend

Has a horn
Is a woven fabric

Is clothing

Is a cylinder

Is a bird

Is a house

Is an animal

Is a root crop

Is a material

Is a weed

Is a cloth

Is a drink

Is a synthetic fibre

Is an insect

Is a lever

Is a weapon

Is a carnivore

Is a primitive craft

Is a shelter

Is a path

Is a kind of meat

Is a floor-covering

Is a fish

Is a projectile

Is an activity

Is a vegetable

Is cutlery

Is a vehicle

Is a pastime

Is a construction

Is a game

Is an automobile

Is a vessel

Is a citrus fruit

Is a dessert

Is an instrument

Is a fowl

Is a building

Is transport

Is seafood

Is a grain

Is an appetiser

Is a weapon

Is a bird

Is a path

Is a tool

Is a tree

Is a delicacy

Is a house

Is a fruit

Is cutlery

Is a bush

Is a flavouring

Is a projectile

Is a shelter

Is an animal

Is food

Is a cylinder

Is a device

Is an object

Is a flower

Is a plant

Is meat

Is a primate

Is a mammal

Is a vegetable

Is metal

Grows in summer

Has a window

Has two hands

Has whiskers

Is nutritious

Has a tail

Is black

Is made of wood

Is for individuals

Has a smell

Has many beds

Is hollow inside

Has long tentacles

Is square

Is eaten smoked

Is very powerful

Has two teams 
HAMPTON

\begin{tabular}{lllll}
\multicolumn{1}{c}{ Word } & True Category & True Property & False Category & False Property \\
\hline Bear & Is a mammal & Has arms & Is poultry & Has a stone \\
Parsnip & Is a root & Has vitamins & Is an insect & Has a lid \\
Lion & Is a carnivore & Has a heart & Is a lever & Has a sail \\
Salmon & Is a delicacy & Has gills & Is furniture & Has a horn \\
Pear & Is a dessert & Has a core & Is clothing & Has a bend \\
Olive & Is an appetiser & Has a stone & Is a barrier & Has springs \\
Raft & Is an object & Has a sail & Is a tuber & Has a point \\
Lamb & Is meat & Has a nose & Is a fish & Has feathers \\
Duck & Is poultry & Has wings & Is a root & Has competitions \\
Knife & Is cutlery & Has an edge & Is a carnivore & Has a core \\
Pavement & Is a path & Has a kerb & Is a bug & Has a head \\
Corkscrew & Is a device & Has a shaft & Is a sport & Has layers \\
Door & Is a construction & Has a catch & Is a creature & Has a roof \\
\hline
\end{tabular}

(Manuscript received December 16, 1983;

revision accepted for publication March 31, 1984.) 